\title{
Mycobacterium tuberculosis Rv3402c Enhances Mycobacterial Survival within Macrophages and Modulates the Host Pro-Inflammatory Cytokines Production via NF-Kappa B/ERK/p38 Signaling
}

\author{
Wu Li, Quanju Zhao, Wanyan Deng, Tian Chen, Minqiang Liu, Jianping Xie*
}

Institute of Modern Biopharmaceuticals, State Key Laboratory Breeding Base of Eco-Environment and Bio-Resource of the Three Gorges Area, Key Laboratory of Ministry of Education Eco-Environment of the Three Gorges Reservoir Region, School of Life Sciences, Southwest University, Chongqing, China

\begin{abstract}
Intracellular survival plays a central role in the pathogenesis of Mycobacterium tuberculosis, a process which depends on an array of virulence factors to colonize and replicate within the host. The $M$. tuberculosis iron regulated open reading frame (ORF) rv3402c, encoding a conserved hypothetical protein, was shown to be up-regulated upon infection in both human and mice macrophages. To explore the function of this ORF, we heterologously expressed the $r v 3402 \mathrm{c}$ gene in the nonpathogenic fast-growing Mycobacterium smegmatis strain, and demonstrated that Rv3402c, a cell envelope-associated protein, was able to enhance the intracellular survival of recombinant $M$. smegmatis. Enhanced growth was not found to be the result of an increased resistance to intracellular stresses, as growth of the Rv3402c expressing strain was unaffected by iron depletion, $\mathrm{H}_{2} \mathrm{O}_{2}$ exposure, or acidic conditions. Colonization of macrophages by M. smegmatis expressing Rv3402c was associated with substantial cell death and significantly greater amount of TNF- $\alpha$ and IL- $1 \beta$ compared with controls. Rv3402cinduced TNF- $\alpha$ and IL-1 $\beta$ production was found to be mediated by NF- $\kappa B$, ERK and p38 pathway in macrophages. In summary, our study suggests that Rv3402c delivered in a live M. smegmatis vehicle can modify the cytokines profile of macrophage, promote host cell death and enhance the persistence of mycobacterium within host cells.
\end{abstract}

Citation: Li W, Zhao Q, Deng W, Chen T, Liu M, et al. (2014) Mycobacterium tuberculosis Rv3402c Enhances Mycobacterial Survival within Macrophages and Modulates the Host Pro-Inflammatory Cytokines Production via NF-Kappa B/ERK/p38 Signaling. PLoS ONE 9(4): e94418. doi:10.1371/journal.pone.0094418

Editor: Shamala Devi Sekaran, University of Malaya, Malaysia

Received September 5, 2013; Accepted March 16, 2014; Published April 10, 2014

Copyright: @ $2014 \mathrm{Li}$ et al. This is an open-access article distributed under the terms of the Creative Commons Attribution License, which permits unrestricted use, distribution, and reproduction in any medium, provided the original author and source are credited.

Funding: This work was supported by National Natural Science Foundation (grant numbers 81371851, 81071316, 81271882), National Megaprojects for Key Infectious Diseases (grant numbers 2008ZX10003-006, 20127X10003-003), New Century Excellent Talents in Universities (grant number NCET-11-0703), Excellent $\mathrm{PhD}$ thesis fellowship of Southwest University (grant numbers kb2010017, ky2011003), the Fundamental Research Funds for the Central Universities (Nos. XDJK2011D006, XDJK2012D011, XDJK2012D007 and XDJK2013D003), Natural Science Foundation Project of CQ CSTC (grant number CSTC 2010BB5002), the Chongqing Municipal Committee of Education for postgraduates excellence program (No. YJG123104), the undergraduates teaching reform program (No. 2011JY052). The funders had no role in study design, data collection and analysis, decision to publish, or preparation of the manuscript.

Competing Interests: The authors have declared that no competing interests exist.

* E-mail: georgex@swu.edu.cn

\section{Introduction}

Tuberculosis (TB), caused by the facultative intracellular pathogen Mycobacterium tuberculosis (Mtb), remains a major cause of morbidity and mortality around the world. Despite intense efforts to mitigate its toll on humanity, TB was still responsible for 1.3 million deaths, and caused 8.6 million new and relapse infections in 2012 (World Health Organization Tuberculosis Data and Statistics, 2013). In addition, the emergence of drug resistant Mtb strains and co-infection with HIV further highlighted the necessity of novel TB medications informed by in-depth understanding of Mtb biology.

Upon internalization by macrophages, Mtb is widely assumed to be confined within the phagosome, a specialized vacuole derived from the plasma membrane. Subsequently, the phagosome matures through fusion with late endosomes and lysosomes, creating an inhospitable environment for invading microorganisms, that includes phagosomal acidification, elevated levels of reactive oxygen intermediates (ROI) and reactive nitrogen intermediates (RNI), charged antimicrobial peptides [1] and iron deprivation [2]. However, Mtb has evolved a spectrum of subversive strategies to survive and thrive inside the macrophages [3].

The success of Mtb can be attributed to an array of virulence factors. These effectors can be classified as (a) enhancing the resistance to host toxic compounds, such as KatG [4], SodC [5,6], AhpC [7,8], (b) blocking phagosomes maturation, such as PtpA $[9,10]$, PknG [11,12], SapM [13,14], and (c) evading apoptosis through NuoG $[15,16,17]$ and SodA $[18,19]$. The identification and characterization of novel virulence factors can enrich our understanding of Mtb biology and facilitate better control measures.

Iron scavenging capacity is critical for pathogen proliferation and pathogenesis within host [20]. The Mtb ORF ro3402c, encoding an IdeR- and iron-repressed gene, was found to be significantly upregulated during Mtb growth under iron-limiting conditions [21]. Interestingly, rv3402c was further shown to be induced in human THP-1 [21] and primary bone marrow-derived murine macrophages [22], suggesting a role in the adaptation to intracellular niches. 
In the following study, we investigated the role of Rv3402c through the construction of recombinant $M$. smegmatis expressing strains. We discovered that Rv3402c was able to enhance $M$. smegmatis' intracellular survival as well as alter the cytokine profile of infected macrophages.

\section{Materials and Methods}

\section{Bacterial strains and growth conditions}

Escherichia coli and M. smegmatis mc $^{2} 155$ strains were provided by the Institute of Modern Biopharmaceuticals. Mtb H37 Rv genomic DNA was provided by Chongqing Pulmonary Hospital. The human leukemic monocyte lymphoma cell line (U-937) was purchased from the Conservation Center in Wuhan University (China), and the murine macrophage cell line (RAW264.7) was a kind gift from Zhiren Zhang [23] (Third Military Medical University, China). Escherichia coli $\mathrm{DH} 5 \alpha$ was routinely grown in LB medium for its use in DNA cloning procedures. M. smegmatis $\mathrm{mc}^{2} 155$ was grown at $37^{\circ} \mathrm{C}$ in Middlebrook (MB) $7 \mathrm{H} 9$ liquid medium or on MB $7 \mathrm{H} 10$ agar supplemented with $0.2 \%(\mathrm{w} / \mathrm{v})$ glucose, $0.5 \%$ (v/v) glycerol and $0.05 \%$ (v/v) Tween 80 . When required, kanamycin $(20 \mu \mathrm{g} / \mathrm{ml})$ or hygromycin $(50 \mu \mathrm{g} / \mathrm{ml})$ was added.

\section{Gene amplification, plasmids construction, and recombinant $M$. smegmatis}

The primers used in this study are listed in Table 1. The pNIT1 plasmid used in this study has been previously described [24]. Briefly, pNIT-Myc was constructed from the pNIT-1 mycobacterial shuttle vector harboring a Myc-tag to the C-terminal of Rv3402c in order to perform Western blot. The full length rv3402c gene was amplified from Mtb H37Rv genomic DNA. Amplified ro3402c gene was then cloned into the pNIT-Myc vector. Constructs for the plasmid pALACE-Rv3402c (at the N terminus) were made by using the similar method as pNIT-Myc-Rv3402c. The $g f p$ gene was excised from pSC301 [25] by digesting with BamHI and ClaI, then cloned into pALACE digested with the same restriction enzymes. The plasmids were electroporated into $M$. smegmatis $\mathrm{mc}^{2} 155$ according to standard procedures [26]. The recombinant $M$. smegmatis strains were selected on $\mathrm{MB} 7 \mathrm{H} 10$ agar containing kanamycin (for pNIT-Myc) or hygromycin (for pALACE). Gene expression was confirmed by PCR, and the recombinant strains were stored at $-70^{\circ} \mathrm{C}$ until further use.

\section{Expression of Rv3402c in M. smegmatis}

The recombinant $M$. smegmatis strains expressing Myc-tagged Rv3402c (MS_Rv3402c) and the empty pNIT-Myc vector (MS_Vec) were cultured until an $\mathrm{OD}_{600}$ 0.6-0.8 was reached. Protein expression was induced with $28 \mathrm{mM}$-caprolactam (Aladdin, China). Total bacterial RNA was isolated using RNAprep Pure Cell/Bacteria Kit (TIANGEN, China) after 16 h induction. An equal amount of total RNA was used as a template for reverse transcriptase (RT)-PGR with ro3402c specific primes and mycobacterium $16 \mathrm{~S}$ rRNA ( $r s B$ gene) specific primers. The RT-PCR products were visualized using a $1 \%$ agarose gel.

For the detection of Myc-tagged Rv3402c bacterial pellets were harvested and disrupted by ultrasonication. Samples were subjected to SDS-PAGE and the Myc-tagged Rv3402c protein was detected by the mouse anti-Myc antibody (TIANGEN, China).

\section{Subcellular fractionation of $M$. smegmatis}

Subcellular fractionation was performed as described by Bashiri et al. [27], with minor modifications. In short, $200 \mathrm{ml}$ of $M$. smegmatis was grown to an $\mathrm{OD}_{600} \sim 0.8$ and was induced with $28 \mathrm{mM} \varepsilon$-caprolactam. Next, the cells were harvested, resuspended in $9 \mathrm{ml}$ lysis buffer (PBS, $1 \mathrm{mM} \mathrm{PMSF}, 0.6 \mathrm{mg} / \mathrm{ml}$ of both DNase and RNase) and then lysed by ultrasonication. Lysates were centrifuged at $3,000 \mathrm{~g}$ for $30 \mathrm{~min}$ to obtain the whole cell lysates (WCL) from the supernatant. WCL was centrifuged at $27,000 \mathrm{~g}$ for $30 \mathrm{~min}$ to obtain the cell wall pellet $(\mathrm{CW})$. The supernatant from the WCL fraction was further centrifuged at $100,000 \mathrm{~g}$ for two hours to separate the cell membrane fraction (pellet, CM) from the soluble fraction (supernatant, SOL). GW and CM fractions were washed once with lysis buffer, re-centrifuged and subsequently re-suspended in $0.5 \mathrm{~mL}$ of lysis buffer. All centrifugation steps were performed at $4{ }^{\circ} \mathrm{C}$.

The above fractionated samples were subjected to SDS-PAGE, with the ratio of protein concentrations at 1:0.36:0.71:0.91 (WCL:CW:CM:SOL). After separation, proteins were transferred to a nitrocellulose membrane. The Myc-tagged Rv3402c protein was detected by the mouse anti-Myc antibody. Native $M$. smegmatis GroEL2, which contains a string of endogenous histidines [28], was detected by an anti-His mouse primary antibody (TIANGEN). The secondary antibody used was goat anti-mouse conjugated to HRP (TIANGEN). The signal was detected using Western Lighting ECL.

\section{Proteinase $\mathrm{K}$ and trypsin sensitivity assays}

Proteinase $\mathrm{K}$ and trypsin sensitivity assays were performed as previously described [29]. Briefly, M. smegmatis harboring pALACE, pALACE-Rv3402c or pALACE-GFP were induced with $0.2 \%$ acetamide for $10 \mathrm{~h}$ and harvested. Cells were washed and mixed with proteinase $\mathrm{K}$ or trypsin at a concentration of $100 \mu \mathrm{g} /$ $\mathrm{ml}$ and incubated at $37^{\circ} \mathrm{C}$ for the indicated times. The reaction was stopped by adding $100 \mathrm{nM}$ PMSF. The samples were dissolved in SDS loading buffer and analyzed by Western blotting using anti-His mouse primary antibody (Abm Inc.) or anti-GFP mouse primary antibody (Boehringer Mannheim Corp.). The secondary antibody used was Alexa Fluor 660 Goat Anti-Mouse IgG (Life technologies Corp.). The signal was detected using Odyssey CLx Infrared Imaging System.

\section{Purification of recombinant Rv3402c protein}

Recombinant Rv3402c (rRv3402c) was expressed in E. coli according to a published protocol [30]. Briefly, the full length ro3402c gene was cloned into the pET-28a(+) vector and the protein was purified by His-tag affinity chromatography on a $\mathrm{Ni}^{2+}$ - nitrilotriacetate column. Purified rRv3402c was dialyzed against PBS (pH 7.2), and then applied to an E-TOXATE Kit (Sigma, USA) to remove any endotoxin contamination prior to filtersterilization, and then stored at $-70^{\circ} \mathrm{C}$ until further use.

\section{Intracellular survival assay}

Suspension cultures of U-937 cells were seeded at $1 \times 10^{6}$ cells per well in 12-well tissue culture plates. Following 48 hours of treatment with $0.1 \mathrm{mg} / \mathrm{ml}$ of phorbol 12-myristate 13-acetate (PMA) (Sigma), U-937 cells were transformed into an adherent state. Cells were infected with $M$. smegmatis transformants at a MOI of $10: 1$ (bacteria-to-U-937 ratio) at $37^{\circ} \mathrm{C}$ in $5 \% \mathrm{CO}_{2}$. Four hours after infection, the remaining bacteria in the culture were removed by washing three times with warm RPMI-1640. RPMI 1640-10\% FCS containing $250 \mathrm{nM} \mathrm{IVN} \mathrm{(Sigma)} \mathrm{in} \mathrm{DMSO} \mathrm{and} \mathrm{hygromycin}$ $\mathrm{B}$ (Roche, USA) $(100 \mu \mathrm{g} / \mathrm{ml})$ were then added. At 6 and $72 \mathrm{~h}$ after infection, the culture supernatants were collected, and stored at $-70^{\circ} \mathrm{C}$. The macrophages were then washed twice and lysed in sterile water containing $0.025 \%$ (w/v) SDS. The lysed macrophages were plated on $\mathrm{MB} 7 \mathrm{H} 10$ agar plates containing 
Table 1. Primers used in this study.

\begin{tabular}{|c|c|}
\hline & Sequence \\
\hline \multicolumn{2}{|l|}{ Cloning primers } \\
\hline pNIT-Rv3402c-For & CGCGGAATTCATGAAGATCCGAAC \\
\hline pNIT-Rv3402c-Rev & AATGGATCCTTCACCGCGCACCT-3 \\
\hline pALACE-Rv3402c-For & GAGGGATCCATGAAGATCCGAACGTT \\
\hline pALACE-Rv3402c-Rev & ACACATATGTCATTCACCGCGCACC \\
\hline pET-28-Rv3402c-For & CGCTGGATCCATGAAGATCCGAAC \\
\hline pET-28-Rv3402c-Rev & GAGAAGCTTTCATTCACCGCGCAC \\
\hline \multicolumn{2}{|l|}{ RT-PCR primers } \\
\hline pNIT-Rv3402c-For & ACCGCTACCTGCTGATG \\
\hline pNIT-Rv3402c-Rev & GGATGGACTCGCGTGTTTG \\
\hline $16 \mathrm{~S}$ rRNA-For & GTAGGGGAAAGCTTTGGGGTGTGG \\
\hline 16 S rRNA-Rev & TCGTCTGTGCTGAAAGAGGTTTACA \\
\hline TNF- $\alpha$-For & CGCTCCCCAAGAAGACAG \\
\hline TNF- $\alpha-\operatorname{Rev}$ & TGAAGAGGACCTGGGAGT \\
\hline IL-1 $\beta$-For & GATGGCTTATTACAGTGGC \\
\hline IL-1 $\beta$-Rev & GACCAGACATCACCAAGC \\
\hline$\beta$-actin-For & CGGCTCCGGCATGTGCAA \\
\hline$\beta$-actin-Rev & ATGTCACGCACGATTTCC \\
\hline
\end{tabular}

kanamycin and the colony forming units (CFUs) were determined as a measure of the intracellular survival of recombinant $M$. smegmatis. The intracellular survival assay of $M$. smegmatis in RAW264.7 cells was performed in a similar manner as described for the U-937 cells above. By comparison, RAW264.7 cells were seeded at $5 \times 10^{5}$ cells per well in 12 -well tissue culture plates. Cells were infected with $M$. smegmatis transformants at a MOI of 10:1 after 24 hours.

\section{In vitro growth and stress assays}

For in vitro growth curves, cultures were inoculated in triplicates with a starting absorbance $\left(\mathrm{OD}_{600}\right)$ of $\sim 0.02$. Broths were incubated at $37^{\circ} \mathrm{C}$ with shaking until an $\mathrm{OD}_{600}$ of $\sim 0.8$ was reached. Twenty-eight micromolar of $\varepsilon$-caprolactam was added to the cultures, and the $\mathrm{OD}_{600}$ was measured at various time points over a $36 \mathrm{~h}$ growth period. To assess the growth curve of recombinant $M$. smegmatis in iron-depleted medium, the recombinant strains were grown with $100 \mu \mathrm{M} 2^{\prime} 2^{\prime}$ dipyridyl [31]. Growth was monitored by determining the $\mathrm{OD}_{600}$ at various time points over $50 \mathrm{~h}$.

To prepare for the $\mathrm{pH}$ stress assays [32], cells were harvested, washed with $7 \mathrm{H} 9(\mathrm{pH} 3$ or 5$)$ and then resuspended to an $\mathrm{OD}_{600}$ of 0.5 in $5 \mathrm{ml} 7 \mathrm{H} 9$ (pH 3 or 5). Cultures were incubated under stress conditions with agitation and $100 \mu \mathrm{l}$ samples were removed for viable cell counts after 0,3 or $6 \mathrm{~h}$. To assess the survival of recombinant $M$. smegmatis after exposure to peroxide stress, the cells were washed and resuspended in $7 \mathrm{H} 9 \mathrm{(pH} \mathrm{5).} \mathrm{Five} \mathrm{milliliters}$ of each M. smegmatis strains, diluted to $\mathrm{OD}_{600}$ of 0.5 in $7 \mathrm{H} 9$ (pH 5), was exposed to $5 \mathrm{mM} \mathrm{H}_{2} \mathrm{O}_{2}$. At 0 and $6 \mathrm{~h}, 100 \mu \mathrm{l}$ samples were removed to determine the viable count. All stress experiments were carried out in triplicates.

\section{Measurement of LDH release from macrophage}

Release of lactate dehydrogenase $(\mathrm{LDH})$ by the U-937 infected with recombinant $M$. smegmatis was measured to evaluate the extent of cytolysis. Culture supernatants were harvested after infection of macrophages with MS_Rv3402c or MS_Vec for 6, 24, 48 or 72 h. LDH activity in culture supernatants was assayed with the CytoTox96 Non-radioactive Cytotoxicity Assay Kit (Promega, USA) as described in the manufacturer's instruction. The percentage of $\mathrm{LDH}$ release was calculated according to published methods [33].

\section{Assay for cytokine production}

U-937 cells were infected at 6 and $72 \mathrm{~h}$ time points, and processed for analysis by a sandwich ELISA. The concentration of cytokines in the culture supernatant was determined using commercially available ELISA kits for tumor necrosis factor alpha (TNF- $\alpha$ ), and interleukin-1 beta (IL-1 $\beta$ ) (eBioscience, USA). All assays were performed as recommended by the manufacturer's protocols.

After infection of macrophages with MS_Rv3402c or MS_Vec for $12 \mathrm{~h}$, total RNA was extracted with the RNAprep Pure Cell/ Bacteria Kit. The DNAase-treated total RNA $(\sim 1 \mu \mathrm{g})$ was transcribed into cDNA with random hexamers using the Transcriptor First Strand cDNA Synthesis Kit (Roche, USA). Semi-quantitative RT-PCR was performed as mentioned above with primers described in Table 1 for the TNF- $\alpha$, IL- $1 \beta$, and $\beta$ actin genes.

\section{Statistical analysis}

Data was analyzed using a Student's two-tailed t-test. Statistical significance was defined as a $p$ - value of 0.05 . Error bars are representative of standard deviation $(\mathrm{SD})$.

\section{Results}

Expression of rv3402c gene in M. smegmatis

The Mtb ORF ro3402c is approximately $1.2 \mathrm{~kb}$ in size and encodes for $45 \mathrm{kDa}$ protein. In this study, we generated two 
recombinant $M$. smegmatis strains to investigate the role of $\mathrm{Rv} 3402 \mathrm{c}$ in host interaction. The MS_Rv3402c strain was engineered to express a Myc-tagged Rv3402c protein from a recombinant pNIT-Myc vector, while the MS_Vec strain harbored the vector alone. Both MS_Rv3402c and MS_Vec, which were grown in MB 7H9 medium in the presence of kanamycin, expressed the aph gene [34]. The semi-RT-PCR results determined that only MS_Rv3402c was able to express the ro3402c gene (Fig. 1A). Western blot analysis using the anti-Myc antibody further confirmed the presence of the expressed $\sim 50-\mathrm{kDa}$ Rv3402c-Myc fusion protein in the cell lysates of the recombinant strain, and its absence in the parental strain (Fig. 1B). These findings indicate that the Rv3402c protein from $M$. tuberculosis was successfully expressed in $M$. smegmatis.

\section{Rv3402c is associated with the mycobacterial cell envelope}

Rv3402c is predicted to be engaged in the biosynthesis of lipopolysaccharide (LPS)-like molecules [21]. Moreover, Rv3402c probably involved in cell process, its sequence shows high similarity to the lipopolysaccharide biosynthesis protein from Bacillus cereus and a putative PLP-dependent enzyme from Rheinheimera sp., which is predicted to be involved in cell wall biogenesis. We therefore tested whether Rv3402c was present in mycobacterial cell wall and cell membrane fractions. We expressed Myc epitope-tagged Rv3402c in M. smegmatis; separated protein extracts into whole cell, cell wall, cytoplasmic, and cell membrane fractions; and analyzed in immunoblot. Although the majority of the Rv3402c proteins were detected in the soluble fraction, small amounts were also seen in the cell wall and cell membrane fractions (Fig. 2A), indicating that Rv3402c is associated with the cell envelope. However, Rv3402c was not detected in culture supernatants and is thus not likely to be secreted extracellularly (data not shown). As controls for fractionation, we show that cytoplasmic heat-shock protein, GroEL2 was detected only in the cytoplasm.

To further confirm the cell surface association of Rv3402c, $M$. smegmatis overexpressing Rv3402c was subjected to proteinase $\mathrm{K}$ and trypsin sensitivity assays as previously described [29,35]. In order to avoid the bias of plasmid pNIT-Myc-Rv3402c, we chose another widely used plasmid pALACE to perform this experiment. As shown in Fig. 2B and C, the His epitope-tagged Rv3402c protein overexpressed by $M$. smegmatis was partly digested by the proteinase $\mathrm{K}$ and trypsin treatment, respectively, even the reaction time prolonged to $30 \mathrm{~min}$. Conversely, the recombinant $M$. smegmatis strain expressing only GFP was protected from digestion. Taken together, although the majority of the Rv3402c proteins are
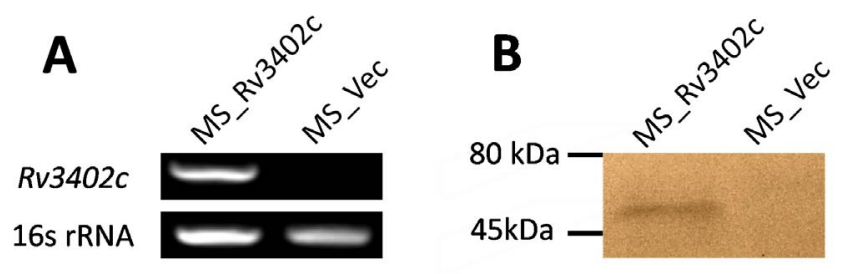

Figure 1. Expression of Rv3402c in recombinant $M$. smegmatis. (A). Ms_Rv3402C and Ms_Vec were grown at $37^{\circ} \mathrm{C}$ in MB $7 \mathrm{H} 9$ liquid medium to an $\mathrm{OD}_{600}$ of 0.6-1.0. Total bacterial RNA was isolated after $16 \mathrm{~h}$ induction and subjected to RT-PCR to detect expression of the $r v 3402 c$ and $r$ rsB genes. (B). Lysates were prepared from bacterial cells that were cultured as in (A) and subjected to Western blot analysis to detect Myc-tagged Rv3402c protein using mouse anti-Myc antibody. doi:10.1371/journal.pone.0094418.g001 localized in the cytoplasm, these results demonstrate that Rv3402c protein is exposed at the mycobacterial cell surface and might gain access to the extracellular environment.

\section{Rv3402c increases the survival of recombinant $M$. smegmatis in macrophages}

Using comparative genomic analysis, no homologus gene could be identified for ro3402c in non-pathogenic species such as $M$. smegmatis, $M$. gilvum and $M$. vanbaalenii. Interestingly, in the attenuated vaccine BCG Pasteur strain, the ro3402c ortholog is disrupted into two fragments. One portion of the fragment is annotated as a pseudogene, the other, a 1053 bp gene known as BCG3472c (99\% identity in DNA sequences). This comparative genomic analysis suggested that $r 03402 c$ might be a novel virulence factor as this gene is not present in non-pathogenic species. To gain further insight as to whether ro3402c is essential for Mtb pathogenesis, we analyzed the intracellular survival of $M$. smegamatis harboring the gene. To achieve this, we infected the mono-layers of U-937 with MS_Vec and MS_Rv3402c as described in the methods section $(\mathrm{MOI}=10: 1)$. The two strains were easily dispersed into single cell suspensions, and the CFU determinations at six hours demonstrated that infection rates were similar in U-937 cells (data not shown). M. smegmatis expressing Rv3402c showed significantly higher bacillary counts in U-937 cells at 48 and 72 hours post infection (Fig. 3A), but differences in survival was only observed at $72 \mathrm{~h}$ in the RAW264.7 cells (Fig. 3B). Despite these differences, these results suggest that the expression of Rv3402c was able to enhance the intracellular survival of $M$. smegmatis in both human and murine macrophages.

\section{Enhanced intracellular survival is not correlated with increased resistance to antimicrobial factors}

To gain further insight as to how the $M$. smegmatis strain harbouring rv3402c confers greater survival in macrophages, we analyzed growth characteristics between the two strains. As seen in Fig. 4A, the two recombinant strains show the same growth kinetics in vitro, suggesting that enhanced survival is likely due to the interaction between mycobacteria and its host. Within the phagosome, the invading microbe is exposed to hostile environment, including iron-deprived conditions, reactive oxygen and reactive nitrogen compounds, and low $\mathrm{pH}$ conditions. As a result, we examined whether $\mathrm{Rv} 3402 \mathrm{c}$ confers resistance to any of these intracellular stresses.

Given that ro3402c is repressed by IdeR and induced by iron limited conditions [21], we explored whether increased survival was due to $M$. smegmatis' resistance to low iron conditions in the macrophage. We compared the ability of the MS_Rv3402c to that of the MS_Vec to grow in MB $7 \mathrm{H} 9$ medium in the presence of 2' $2^{\prime}$ dipyridyl, an iron chelator. No significant differences were observed between the MS_Rv3402c and the empty vector strain in the presence of $100 \mu \mathrm{M} 2^{\prime} 2^{\prime}$ dipyridyl (Fig. 4B).

To assess acid sensitivity, growth of the two recombinant $M$. smegmatis strains was monitored in $\mathrm{MB} 7 \mathrm{H} 9$ at $\mathrm{pH} 3.0$ and 5.0, and was compared at three time points during a six hours incubation. No growth differences were observed between these two strains at any time (Fig. 4C). Similarly, susceptibility of the two recombinant strains to ROI was observed following six hours exposure to $5 \mathrm{mM}$ $\mathrm{H}_{2} \mathrm{O}_{2}$. The percentage of survival of MS_Vec and MS_Rv3402c was $123.3 \%$ and $116.7 \%$, respectively, with no difference observed between the two recombinants (Fig. 4D). Taken together, these results suggest that the increased survival of MS_Rv3402c in the macrophage cannot be attributed to the increased resistance to any of the tested stresses. 
A

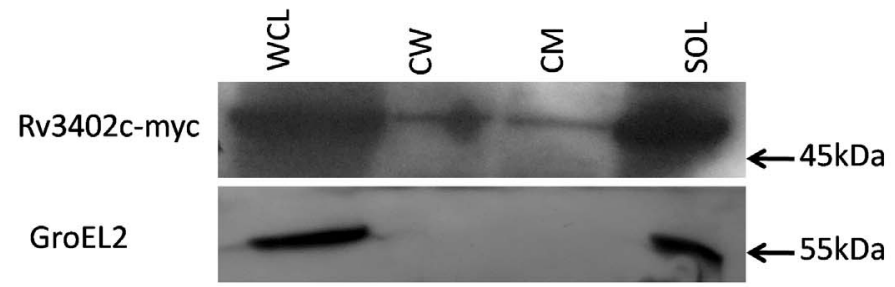

B

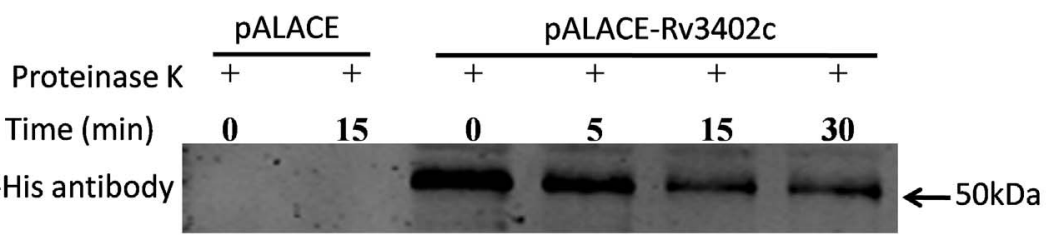

Anti-GFP antibody

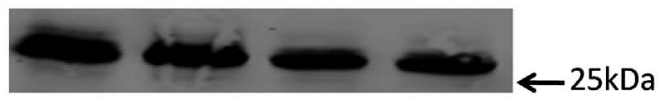

C

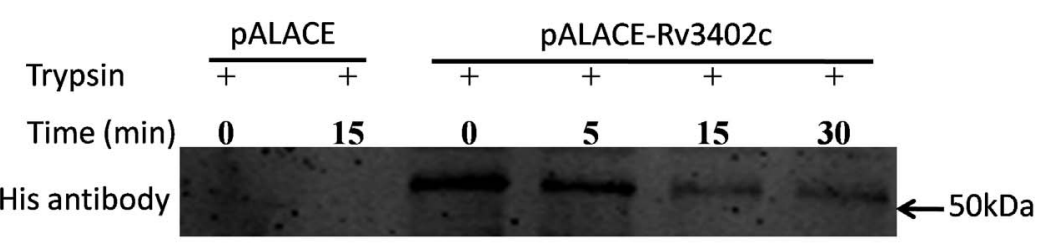

Anti-GFP antibody

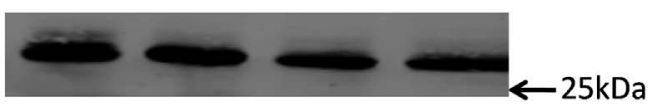

Figure 2. Rv3402c is a cell envelope-associated protein. (A). Subcellular fractionation of $M$. smegmatis induced to express Rv3402c-myc showed localization to the cell wall and membrane fractions. Subcellular fractions were separated by SDS-PAGE and proteins were detected with an anti-Myc antibody. Native GroEL2 was detected as a cytoplasmic control. WCL, whole cell lysates ( $66 \mu \mathrm{g}$ total protein); CW, cell wall fraction (24 $\mu \mathrm{g}$ total protein); CM, cell membrane fraction (47 $\mu \mathrm{g}$ total protein); SOL, soluble fraction (60 $\mu \mathrm{g}$ total protein). (B and C). M. smegmatis transformed with pALACE, pALACE-GFP or pALACE-Rv3402c were incubated with proteinase $\mathrm{K}$ or trypsin at different time points. Whole-cell lysates obtained by the recombinant bacteria were separated on SDS-PAGE. Immunoblots were performed using anti-His antibodies and anti-GFP antibodies. Similar results were obtained in two independent experiments.

doi:10.1371/journal.pone.0094418.g002
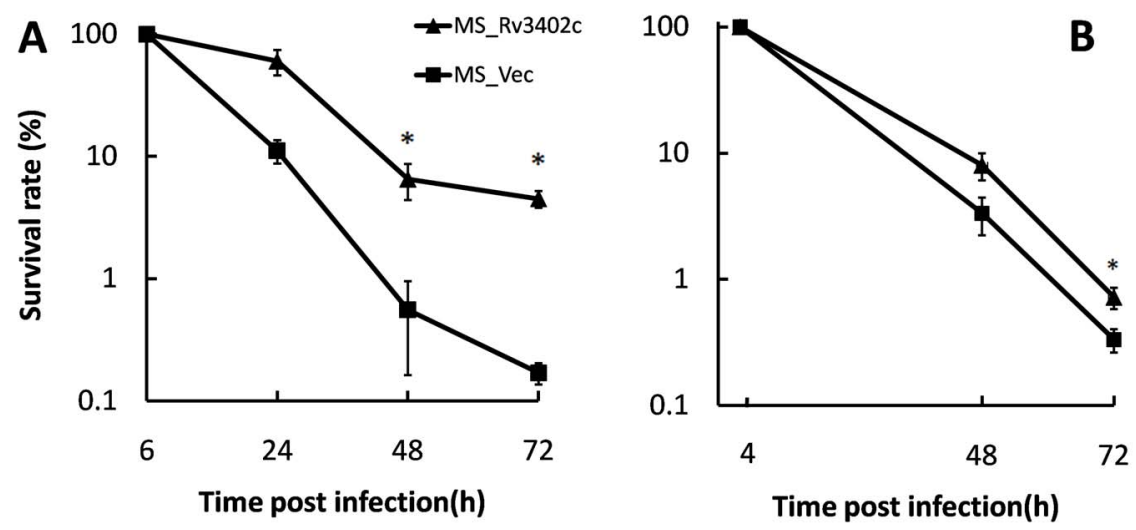

Figure 3. Intracellular survival of recombinant $M$. smegmatis in macrophages. U-937 cells (A) and RAW267.4 cells (B) were infected with MS_Vec and MS_Rv3402c as described in the methods section, respectively. Aliquots of infected cells were lysed with $0.025 \%$ SDS at indicated times, and serial dilutions were plated on $7 \mathrm{H} 10$ agar plates containing kanamycin. Recovered CFUs were enumerated after the incubation for 3-4 days at $37^{\circ} \mathrm{C}$. Numbers of intracellular bacteria are shown as a percentage of the numbers detected at $\mathrm{t}=6 \mathrm{~h}$ (U-937) or $4 \mathrm{~h}$ (RAW267.4) (\% survival). Data are shown as means \pm SD of triplicate wells. Similar results were obtained in three independent experiments. doi:10.1371/journal.pone.0094418.g003 

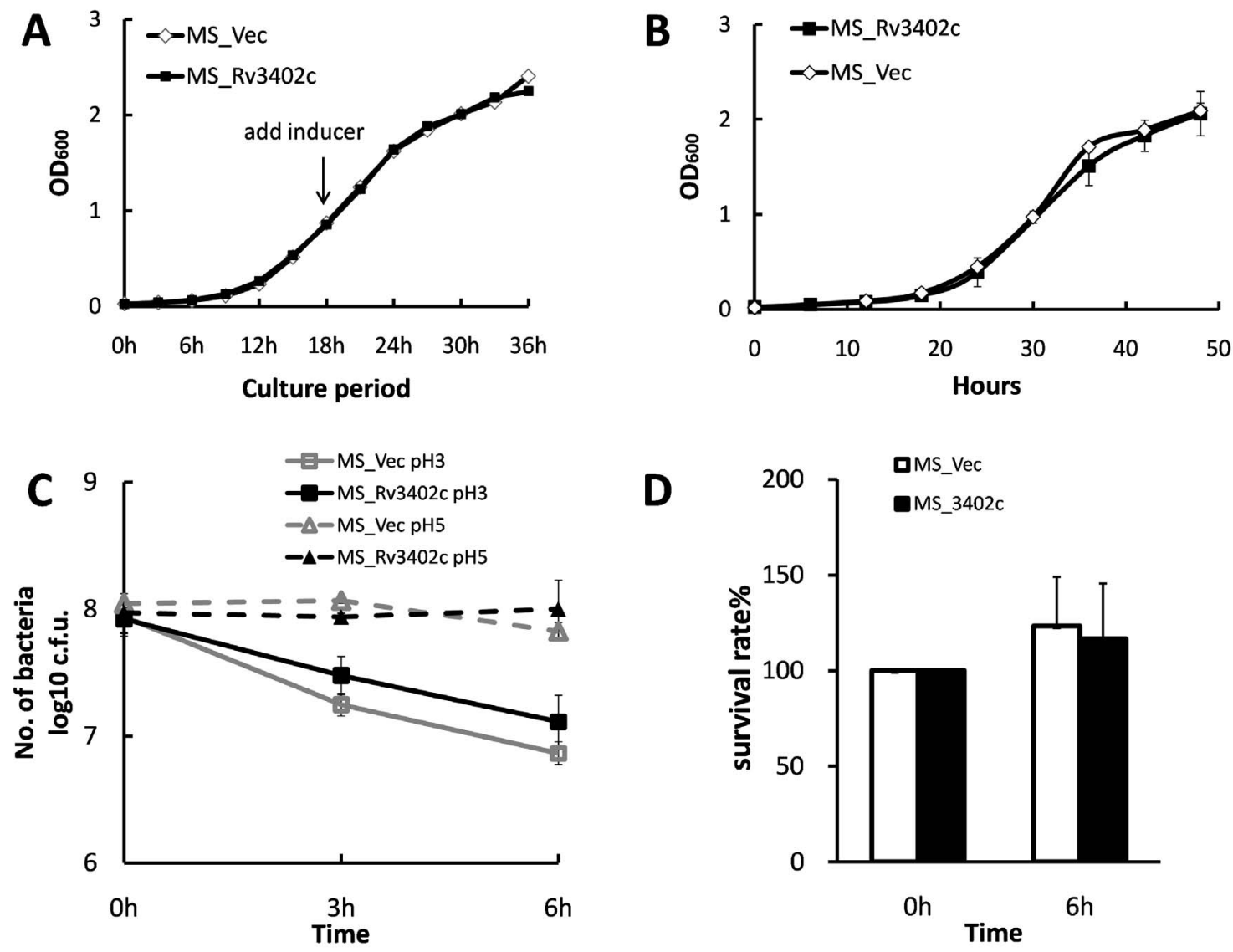

Figure 4. Growth of MS_Vec and MS_Rv3402c under stress conditions. (A). Growth of MS-Vec and MS-Rv3402c at $37^{\circ} \mathrm{C}$ in $\mathrm{MB} 7 \mathrm{H} 9$ liquid medium was monitored by determining $\mathrm{OD}_{600}$ at intervals of $3 \mathrm{~h}$. (B). Growth curve of MS-Vec and MS-Rv3402c in iron-depleted medium. Two recombinant $M$. smegmatis were grown in $\mathrm{MB} 7 \mathrm{H} 9$ medium supplemented with $100 \mu \mathrm{M} 2^{\prime} 2^{\prime}$ dipyridyl. The growth of the strains were monitored by measuring $\mathrm{OD}_{600}$ at intervals of $6 \mathrm{~h}$. (C). In vitro growth of MS-Vec and MS-Rv3402c at different $\mathrm{pH}$. The bacteria were collected by centrifugation and resuspended to an $\mathrm{OD}_{600}$ of 0.5 in $5 \mathrm{ml} \mathrm{MB} 7 \mathrm{H} 9$ ( $\mathrm{pH} 5$ or 3). All cultures were again incubated at $37^{\circ} \mathrm{C}$ and 0.1 ml removed for viable count enumeration after 0,3 and 6 h. (D). Survival of recombinant M. smegmatis after exposure to hydrogen peroxide. Aliquots $(5 \mathrm{ml})$ of cultures at $\mathrm{OD}_{600}$ of 0.5 were exposed to $5 \mathrm{mM}$ hydrogen peroxide $\left(\mathrm{H}_{2} \mathrm{O}_{2}\right)$ for $6 \mathrm{~h}$ at $37^{\circ} \mathrm{C}$. The cultures were serially diluted and plated onto $\mathrm{MB} 7 \mathrm{H} 10$ plates and the colonies counted after $3-4$ days of incubation at $37^{\circ} \mathrm{C}$.

doi:10.1371/journal.pone.0094418.g004

The effect of the recombinant $M$. smegmatis expressing Rv3402c on the viability of macrophages

One of the destinies of macrophages infected with Mtb is cell death due to the pathogen's ability to alter host cell signaling pathways. To test the effect of Rv3402c on macrophage viability, U-937 cell lines were infected by recombinant $M$. smegmatis containing Rv3402c or the control strain. The amount of $\mathrm{LDH}$ was measured in the culture supernatants to quantify cell lysis. Six hours following infection with MS_Rv3402c, there was a significant release of $\mathrm{LDH}$ that increased to a range of $49.8 \%$ and $50.4 \%$ over 72 hours in culture (Fig. 5). In contrast, the percentage of LDH release did not exceed $31 \%$ in cultures infected with MS_Vec. These results show that infection with the $M$. smegmatis strain harbouring ro3402c was able to induce macrophage lysis.

\section{Rv3402c upregulates TNF- $\alpha$ and IL-1 $\beta$ production}

To explore the potential role of Rv3402c in subverting the innate immune response, we investigated the levels of proinflammatory cytokines upon infection of U-937 with MS_Vec and MS_Rv3402c. Macrophages infected with MS_Rv3402c secreted significantly higher levels of TNF- $\alpha$ and IL-1 $\beta$ than the

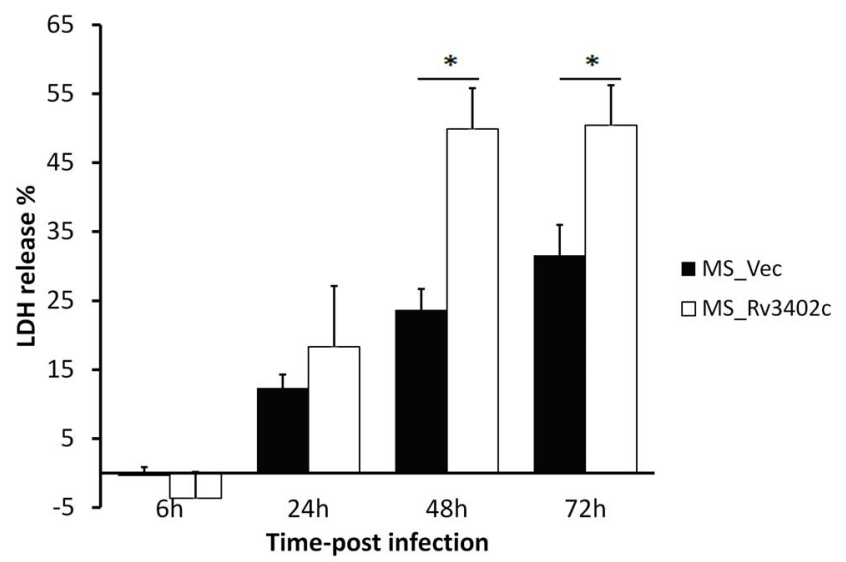

Figure 5. Assay of cell death in macrophages infected with recombinant $M$. Smegmatis. Culture supernatants were collected from mono-layers of U-937 infected at a MOI of 10:1 with MS Vec or MS_Rv3402c and the release of LDH as a measure of macrophage cell death was estimated at various time points. Data are shown as means \pm SD of triplicate wells. Similar results were obtained in three independent experiments.

doi:10.1371/journal.pone.0094418.g005 
MS_Vec infected cells (Fig. 6A and C). To confirm these observations, the pro-inflammatory cytokine mRNA expression was determined using semi-RT-PCR. Compared to the MS_Vec, mRNA levels of TNF- $\alpha$ (Fig. 6B) and IL-1 $\beta$ (Fig. 6D) were significant upregulated in macrophages after 12 hours of infection with MS_Rv3402c. Similar results were obtained when U-937 cells were stimulated with rRv3402c (Fig. 7A and B). No difference was found for the levels of other inflammatory proteins, including IL-6, IL-10, and IL-12 p40 (data not shown). Collectively, our data suggests that $\mathrm{Rv} 3402 \mathrm{c}$ might play a role in modulating the proinflammatory cytokine production of macrophages infected with M. smegmatis.

\section{Rv3402c robustly activates macrophage NF- $\mathrm{KB}$, ERK and p38 signaling}

$\mathrm{NF}-\kappa \mathrm{B}$ is a major transcription factor responsible for the transcription of TNF- $\alpha[36,37,38]$. ERK $1 / 2$ activation is essential for TNF- $\alpha$ production by macrophages $[39,40]$, and the MAPK pathways are crucial for macrophage signaling during mycobacterial infection $[39,41]$. The increased TNF- $\alpha$ and IL- $1 \beta$ mRNA and protein expression (Fig. 6) raised the speculation that the activation of these signaling pathways might be altered within macrophages infected with MS_Rv3402c. To test this possibility, pharmacological inhibition experiments were used to confirm the requirement for $\mathrm{NF}-\kappa \mathrm{B}, \mathrm{ERK}$ and $\mathrm{p} 38$ activities in the production of TNF- $\alpha$ and IL-1 $\beta$ in macrophages infected with MS_Rv3402c. MS_Rv3402c-induced TNF- $\alpha$ expression and protein production were significantly inhibited in U-937 cells pretreated with the specific NF-кB inhibitor TPCK, I kappa B-protease inhibitor [42], the ERK 1/2 inhibitor PD 98,059, and the p38 inhibitor SB 202190. However, IL-1 $\beta$ mRNA and protein expression were only inhibited by NF- $\mathrm{KB}$ specific inhibitor and the ERK $1 / 2$ inhibitor (Fig. 7C and D). After stimulation with $r R v 3402 c$ for the time points indicated, similar results were obtained (Fig. 7E). Pretreatment of U-937 cells with the NF- $\mathrm{BB}$ inhibitor, the ERK 1/2 inhibitor and the p38 inhibitor significantly reduced the production of TNF- $\alpha$ in a dose-dependent manner (Fig. 7E). Taken together, these results imply that the $\mathrm{NF}-\kappa \mathrm{B}$, ERK and p38 pathways are required for Rv3402c-induced TNF- $\alpha$ and IL-1 $\beta$ production by macrophages.

\section{Discussion}

The persistence of pathogenic mycobacteria within macrophages is in part due to the bacteria's ability to actively manipulate host signaling. Several mycobacterial secreted virulence factors have been well defined in the process of inhibiting phagosomal maturation, and such knowledge will unveil previously unknown host signaling pathways involved in the innate immunity against Mtb infection, which will lead to the development of more effective vaccines and drugs.

Our data suggests that the Rv3402c protein might play a role in intracellular survival of recombinant $M$. smegmatis by modulating the host innate immune response. M. smegmatis, a non-pathogenic mycobacterium species, does not multiply and is readily killed by the macrophage, enabling this bacteria to be an ideal surrogate for the identification of virulence factors implicated in intracellular survival $[26,32,33,43,44,45,46,47,48,49]$. We found that $M$. smegmatis recombinant strain expressing Rv3402c showed enhanced survival both in the U-937 and the RAW264.7 cultures in vitro and impaired the viability of U-937. These results were not observed in the $M$. smegmatis strain containing the vector only. Since these two recombinant strains show the same growth kinetics in axenic culture or other stress conditions, the enhanced intracellular survival in macrophages of MS_Rv3402c was thought to be the result of the protein's interference with the innate immune response. Differences in viability between the U-937 and RAW264.7 cells infected with MS_3402c was possibly due to the inherent differences between mouse and human macrophages. For example, the Mtb $p t p A$ deletion mutant was described to have reduced virulence in THP-1 cells $[9,10]$. However, the same mutant was not found to have reduced survival in a mouse infection model [50]. Thus, definitive identification of the role of Rv3402c in these two models will require further functional studies which are beyond the scope of this study.

Despite recent evidence of the role of $r 03402 c$ in the interaction of recombinant $M$. smegmatis and host cells, the function of

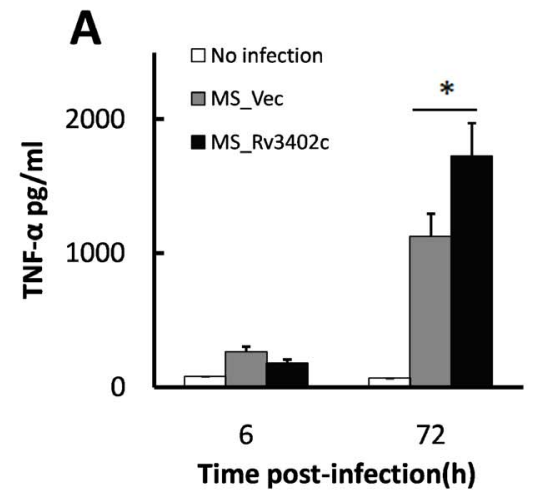

B

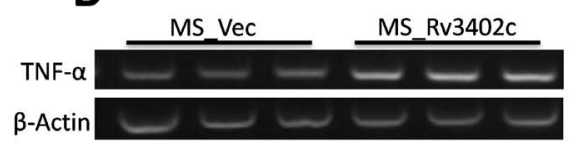

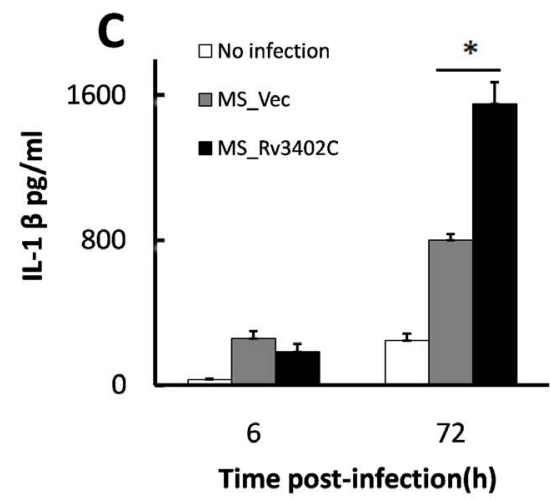

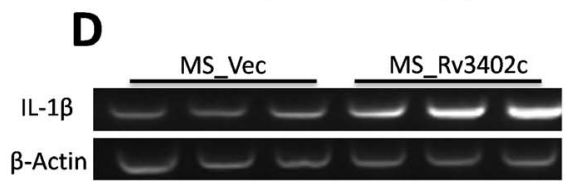

Figure 6. Rv3402c induces the secretion of TNF- $\alpha$ and IL-1 $\beta$ in infected macrophages. Culture supernatants were harvested after 6 or $72 \mathrm{~h}$ of infection and the concentrations of TNF- $\alpha(A)$ and IL-1 $\beta(C)$ were determined. Cells were harvested after 12 h of infection and semi-quantitative RTPCR analysis of TNF- $\alpha$ (B) and IL-1 $\beta$ (D) mRNA level was performed. Each three lanes in the RT-PCR analysis are replicates of a single time point. Similar results were obtained in three independent experiments.

doi:10.1371/journal.pone.0094418.g006 

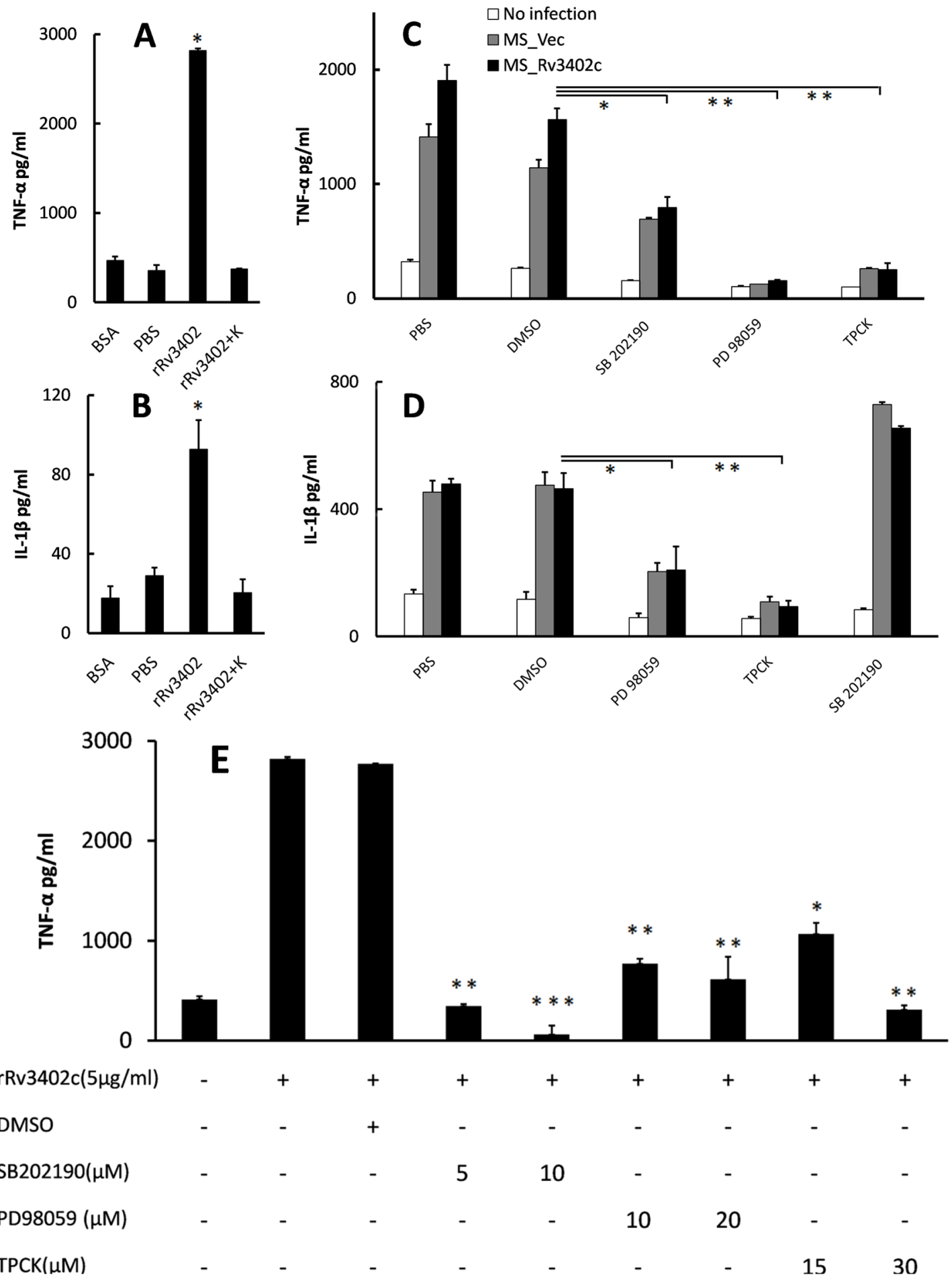

Figure 7. Rv3402c alters the activation levels of NF-KB, ERK and p38 in macrophages infected with $M$. smegmatis. Culture supernatants were harvested after $12 \mathrm{~h}$ of treatment with $\mathrm{rRv} 3402 \mathrm{C}$ at the concentration of $5 \mu \mathrm{g} / \mathrm{ml}$ and the concentrations of TNF- $\alpha$ (A) and IL-1 $\beta$ (B) were determined. U-937 cells were pre-treated with $30 \mu \mathrm{M}$ TPCK (a NF-kB inhibitor) or with $20 \mu \mathrm{M}$ PD 98059 (an ERK1/2 inhibitor) or with $10 \mu \mathrm{M}$ SB 202190 (a p38 inhibitor). Treatment with DMSO served as a control for the inhibitor treatments. After $1 \mathrm{~h}$, the macrophages were infected with MS_Vec at an $\mathrm{MOI}$ of 10 or treated with rRv3402c at the concentration of $5 \mu \mathrm{g} / \mathrm{ml}$. Protease K (K) used to digest the recombinant protein. Culture supernatants were harvested after $24 \mathrm{~h}$ of infection and the concentrations of TNF- $\alpha(C, E)$ and IL-1 $\beta$ (D) were determined. (A), (B) and (E) are performed with recombinant protein; $(C)$ and $(D)$ are performed with $M$. smegmatis strains. The data are representative of two independent experiments. doi:10.1371/journal.pone.0094418.g007

Rv3402c remains unknown. According to the TubercuList website (http://tuberculist.epfl.ch/index.html), rv3402c encodes a conserved hypothetical protein. Rv3402c is predicted to be engaged in the biosynthesis of lipopolysaccharide (LPS)-like molecules, and its sequence shows $>40 \%$ sequence similarity to three types of enzymes: an aminotransferase, a dehydratase and an enzyme 
involved in perosamine/O-antigen biosynthesis [21]. Moreover, using the NCBI BLAST server we found that ro3402c encodes a protein with high sequence similarity to the lipopolysaccharide biosynthesis protein from Bacillus cereus and a putative PLPdependent enzyme from Rheinheimera sp., which is predicted to be involved in cell wall biogenesis. We therefore tested whether Rv3402c was altered the cell surface properties. We found that the overexpression in M. smegmatis of Rv3402c, which is found in the cell envelope of mycobacteria, does not impact on colony morphology (data not shown). Furthermore, bacterial fatty acids compositions of $M$. smegmatis was not affected by the overexpression of Rv3402c protein (data not shown).

Although the physiologic function of Rv3402c is poorly understood, based on the subcellular fractionation analysis in this study, this protein deserved to be investigated for its role as a membrane- or cell wall-associated components. In order to avoid the bias of plasmid pNIT-Myc-Rv3402c, we chose another widely used plasmid pALACE to perform proteinase $\mathrm{K}$ and trypsin sensitivity assays. These assays performed on live recombinant mycobacterial strains revealed that His-tagged Rv3402c protein is partly exposed on the surface of mycobacteria. Not surprisingly, there are many proteins detected both in the cytoplasm and cell envelope, such as Rv2224c [51], Rv0132c [27], Eis [52] PknI [53], et al. Together, these results provide evidence that the Rv3402c protein is present on the surface of mycobacteria and may be available for interaction with host components.

TNF- $\alpha$, a critical pro-inflammatory cytokine, is essential for host protective immunity to contain $M$. tuberculosis infection $[54,55]$. Compared to pathogenic mycobacteria, M. smegmatis is a potent inducer of TNF- $\alpha$ in macrophages [56], consistent with the elevated TNF- $\alpha$ levels in the supernatant of macrophages infection with MS_Vec instead of the non-infected groups. Elevated levels of TNF- $\alpha$ was regarded as one culprit for Mtb persistence and virulence within human macrophages [45]. Previous studies have shown that mycobacterial components can enhance the production of TNF- $\alpha$ in monocytes/macrophages [45,57,58,59]. Other

\section{References}

1. Flannagan RS, Cosio G, Grinstein S (2009) Antimicrobial mechanisms of phagocytes and bacterial evasion strategies. Nat Rev Microbiol 7: 355-366.

2. De Voss JJ, Rutter K, Schroeder BG, Su H, Zhu Y, et al. (2000) The salicylatederived mycobactin siderophores of Mycobacterium tuberculosis are essential for growth in macrophages. Proc Natl Acad Sci U S A 97: 1252-1257.

3. Li W, Xie J (2011) Role of mycobacteria effectors in phagosome maturation blockage and new drug targets discovery. J Cell Biochem 112(10): 2688-2693.

4. Ng VH, Cox JS, Sousa AO, MacMicking JD, McKinney JD (2004) Role of KatG catalase-peroxidase in mycobacterial pathogenesis: countering the phagocyte oxidative burst. Mol Microbiol 52: 1291-1302.

5. Fridovich I (1995) Superoxide radical and superoxide dismutases. Annu Rev Biochem 64: 97-112.

6. Spagnolo L, Toro I, D’Orazio M, O’Neill P, Pedersen JZ, et al. (2004) Unique features of the sodC-encoded superoxide dismutase from Mycobacterium tuberculosis, a fully functional copper-containing enzyme lacking zinc in the active site. J Biol Chem 279: 33447-33455.

7. Wilson T, de Lisle GW, Marcinkeviciene JA, Blanchard JS, Collins DM (1998) Antisense RNA to $\operatorname{ahp} C$, an oxidative stress defence gene involved in isoniazid resistance, indicates that $\mathrm{AhpC}$ of Mycobacterium bovis has virulence properties. Microbiology 144 (Pt 10): 2687-2695.

8. Master SS, Springer B, Sander P, Boettger EC, Deretic V, et al. (2002) Oxidative stress response genes in Mycobacterium tuberculosis: role of ahpC in resistance to peroxynitrite and stage-specific survival in macrophages. Microbiology 148: 3139-3144.

9. Bach H, Papavinasasundaram KG, Wong D, Hmama Z, Av-Gay Y (2008) Mycobacterium tuberculosis virulence is mediated by PtpA dephosphorylation of human vacuolar protein sorting 33B. Cell Host Microbe 3: 316-322.

10. Wong D, Bach H, Sun J, Hmama Z, Av-Gay Y (2011) Mycobacterium tuberculosis protein tyrosine phosphatase (PtpA) excludes host vacuolar- $\mathrm{H}^{+}$-ATPase to inhibit phagosome acidification. Proc Natl Acad Sci U S A 108: 19371-19376.

11. Cowley S, Ko M, Pick N, Chow R, Downing KJ, et al. (2004) The Mycobacterium tuberculosis protein serine/threonine kinase PknG is linked to cellular glutamate/ studies have shown that virulent growth of $\mathrm{Mtb}$ in human monocytes [60,61] or alveolar macrophages [62] is associated with enhanced secretion of TNF, as well as cytotoxicity. This evidence may provide insight into the role of TNF in cytolysis and the enhanced survival of the $M$. smegmatis strain expressing Rv3402c. Finally, through the pharmacological inhibition experiments, we found that the NF- $\kappa \mathrm{B}, \mathrm{ERK}$ and p38 pathways are required for Rv3402c-induced TNF- $\alpha$ expression by macrophages. However, only the NF- $\kappa \mathrm{B}$ and ERK1/2 pathways are required for Rv3402cinduced IL-1 $\beta$ expression and protein production. It is our observation that Rv3402c disrupts host signal transduction in an alternative manner, which ultimately enables the bacteria to subvert the host immune response.

In summary, the present study suggests that the expression of the Rv3402c protein in $M$. smegmatis provides the bacteria specific properties that enhance intracellular persistence, decrease viability and modify the pro-inflammatory cytokine response of macrophages. This might provide novel information in the complex interplay between the host and Mtb. A greater understanding of the effect of Rv3402c on host signaling pathways will enhance our knowledge of Mtb pathogenesis. Further experiments such as using Mtb knockout mutant to determine the precise function of Rv3402c during infection are worthwhile.

\section{Acknowledgments}

We would like to thank Dr. Yossef Av-Gay for providing facilities with the proteinase $\mathrm{K}$ and trypsin sensitivity assays. We thank Melissa RichardGreenblatt (UBC, Canada) for her help with critical reading and revision of this manuscript.

\section{Author Contributions}

Conceived and designed the experiments: WL JX. Performed the experiments: WL QZ WD TC ML. Analyzed the data: WL. Contributed reagents/materials/analysis tools: WL QZ WD ML. Wrote the paper: WL JX.

glutamine levels and is important for growth in vivo. Mol Microbiol 52: 16911702 .

12. Walburger A, Koul A, Ferrari G, Nguyen L, Prescianotto-Baschong C, et al. (2004) Protein kinase G from pathogenic mycobacteria promotes survival within macrophages. Science 304: 1800-1804.

13. Vergne I, Chua J, Lee HH, Lucas M, Belisle J, et al. (2005) Mechanism of phagolysosome biogenesis block by viable Mycobacterium tuberculosis. Proc Natl Acad Sci U S A 102: 4033-4038.

14. van der Wel N, Hava D, Houben D, Fluitsma D, van Zon M, et al. (2007) M. tuberculosis and $M$. leprae translocate from the phagolysosome to the cytosol in myeloid cells. Cell 129: 1287-1298.

15. Velmurugan K, Chen B, Miller JL, Azogue S, Gurses S, et al. (2007) Mycobacterium tuberculosis nuoG is a virulence gene that inhibits apoptosis of infected host cells. PLoS Pathog 3: e110.

16. Miller JL, Velmurugan K, Cowan MJ, Briken V (2010) The type I NADH dehydrogenase of Mycobacterium tuberculosis counters phagosomal $\mathrm{NOX}_{2}$ activity to inhibit TNF-alpha-mediated host cell apoptosis. PLoS Pathog 6: e1000864.

17. Blomgran R, Desvignes L, Briken V, Ernst JD (2012) Mycobacterium tuberculosis inhibits neutrophil apoptosis, leading to delayed activation of naive CD4 T cells. Cell Host Microbe 11: 81-90.

18. Braunstein M, Espinosa BJ, Chan J, Belisle JT, Jacobs WR Jr (2003) SecA2 functions in the secretion of superoxide dismutase $\mathrm{A}$ and in the virulence of Mycobacterium tuberculosis. Mol Microbiol 48: 453-464.

19. Kurtz S, McKinnon KP, Runge MS, Ting JP, Braunstein M (2006) The SecA2 secretion factor of Mycobacterium tuberculosis promotes growth in macrophages and inhibits the host immune response. Infect Immun 74: 6855-6864.

20. Li W, He J, Xie L, Chen T, Xie J (2013) Comparative genomic insights into the biosynthesis and regulation of mycobacterial siderophores. Cell Physiol Biochem 31: $1-13$.

21. Gold B, Rodriguez GM, Marras SA, Pentecost M, Smith I (2001) The Mycobacterium tuberculosis IdeR is a dual functional regulator that controls transcription of genes involved in iron acquisition, iron storage and survival in macrophages. Mol Microbiol 42: 851-865. 
22. Schnappinger D, Ehrt S, Voskuil MI, Liu Y, Mangan JA, et al. (2003) Transcriptional adaptation of Mycobacterium tuberculosis within macrophages: Insights into the phagosomal environment. J Exp Med 198: 693-704.

23. Zhang Z, Zhang ZY, Schittenhelm J, Wu Y, Meyermann R, et al. (2011) Parenchymal accumulation of $\mathrm{CD} 163^{+}$macrophages/microglia in multiple sclerosis brains. J Neuroimmunol 237: 73-79.

24. Pandey AK, Raman S, Proff R, Joshi S, Kang GM, et al. (2009) Nitrile-inducible gene expression in mycobacteria. Tuberculosis (Edinb) 89: 12-16.

25. Cowley SC, Av-Gay Y (2001) Monitoring promoter activity and protein localization in Mycobacterium spp. using green fluorescent protein. Gene 264: $225-231$.

26. Li JM, Li N, Zhu DY, Wan LG, He YL, et al. (2008) Isocitrate lyase from Mycobacterium tuberculosis promotes survival of Mycobacterium smegmatis within macrophage by suppressing cell apoptosis. Chin Med J (Engl) 121: 1114-1119.

27. Bashiri G, Perkowski EF, Turner AP, Feltcher ME, Braunstein M, et al. (2012) Tat-Dependent Translocation of an F(420)-Binding Protein of Mycobacterium tuberculosis. PLoS One 7: e45003.

28. Rengarajan J, Murphy E, Park A, Krone CL, Hett EC, et al. (2008) Mycobacterium tuberculosis Rv2224c modulates innate immune responses. Proc Natl Acad Sci USA 105: 264-269.

29. Chaturvedi R, Bansal K, Narayana Y, Kapoor N, Sukumar N, et al. (2010) The multifunctional PE_PGRS11 protein from Mycobacterium tuberculosis plays a role in regulating resistance to oxidative stress. J Biol Chem 285: 30389-30403.

30. Ji L, Long Q, Yang D, Xie J (2011) Identification of mannich base as a novel inhibitor of Mycobacterium tuberculosis isocitrate by high-throughput screening. Int J Biol Sci 7: 376-382.

31. Reddy PV, Puri RV, Khera A, Tyagi AK (2011) Iron storage proteins are essential for the survival and pathogenesis of Mycobacterium tuberculosis in THP-1 macrophages and the guinea pig model of infection. J Bacteriol 194: 567-575.

32. Pelosi A, Smith D, Brammananth R, Topolska A, Billman-Jacobe H, et al. (2012) Identification of a novel gene product that promotes survival of Mycobacterium smegmatis in macrophages. PLoS One 7: e31788.

33. Daim S, Kawamura I, Tsuchiya K, Hara H, Kurenuma T, et al. (2011) Expression of the Mycobacterium tuberculosis PPE37 protein in Mycobacterium smegmatis induces low tumour necrosis factor alpha and interleukin 6 production in murine macrophages. J Med Microbiol 60: 582-591.

34. Stover CK, de la Cruz VF, Fuerst TR, Burlein JE, Benson LA, et al. (1991) New use of BCG for recombinant vaccines. Nature 351: 456-460.

35. Dong D, Wang D, Li M, Wang H, Yu J, et al. (2012) PPE38 modulates the innate immune response and is required for Mycobacterium marinum virulence. Infect Immun 80: 43-54.

36. Collart MA, Baeuerle P, Vassalli P (1990) Regulation of tumor necrosis factor alpha transcription in macrophages: involvement of four kappa B-like motifs and of constitutive and inducible forms of NF-kappa B. Mol Cell Biol 10: 1498-1506.

37. Kuprash DV, Udalova IA, Turetskaya RL, Kwiatkowski D, Rice NR, et al. (1999) Similarities and differences between human and murine TNF promoters in their response to lipopolysaccharide. J Immunol 162: 4045-4052.

38. Baeuerle PA, Henkel T (1994) Function and activation of NF-kappa B in the immune system. Annu Rev Immunol 12: 141-179.

39. Yadav M, Roach SK, Schorey JS (2004) Increased mitogen-activated protein kinase activity and TNF-alpha production associated with Mycobacterium smegmatis- but not Mycobacterium avium-infected macrophages requires prolonged stimulation of the calmodulin/calmodulin kinase and cyclic AMP/protein kinase A pathways. J Immunol 172: 5588-5597.

40. Jo EK, Yang CS, Choi CH, Harding CV (2007) Intracellular signalling cascades regulating innate immune responses to Mycobacteria: branching out from Tolllike receptors. Cell Microbiol 9: 1087-1098.

41. Pathak SK, Bhattacharyya A, Pathak S, Basak C, Mandal D, et al. (2004) Tolllike receptor 2 and mitogen- and stress-activated kinase 1 are effectors of Mycobacterium avium-induced cyclooxygenase-2 expression in macrophages. J Biol Chem 279: 55127-55136.

42. Kengatharan M, De Kimpe SJ, Thiemermann C (1996) Analysis of the signal transduction in the induction of nitric oxide synthase by lipoteichoic acid in macrophages. Br J Pharmacol 117: 1163-1170.

43. Kuprash DV, Alimzhanov MB, Tumanov AV, Anderson AO, Pfeffer K, et al. (1999) TNF and lymphotoxin beta cooperate in the maintenance of secondary lymphoid tissue microarchitecture but not in the development of lymph nodes. J Immunol 163: 6575-6580.

44. Huang Y, Wang Y, Bai Y, Wang ZG, Yang L, et al. (2010) Expression of PE_PGRS 62 protein in Mycobacterium smegmatis decrease mRNA expression of proinflammatory cytokines IL-1 $\beta$, IL-6 in macrophages. Mol Cell Biochem 340: 223-229.

45. Dheenadhayalan V, Delogu G, Brennan MJ (2006) Expression of the PE_PGRS 33 protein in Mycobacterium smegmatis triggers necrosis in macrophages and enhanced mycobacterial survival. Microbes Infect 8: 262-272.

46. Nair S, Ramaswamy PA, Ghosh S, Joshi DC, Pathak N, et al. (2009) The PPE18 of Mycobacterium tuberculosis interacts with TLR2 and activates IL-10 induction in macrophage. J Immunol 183: 6269-6281.

47. Delogu G, Pusceddu C, Bua A, Fadda G, Brennan MJ, et al. (2004) Rv1818cencoded PE_PGRS protein of Mycobacterium tuberculosis is surface exposed and influences bacterial cell structure. Mol Microbiol 52: 725-733.

48. Lagier B, Pelicic V, Lecossier D, Prod'hom G, Rauzier J, et al. (1998) Identification of genetic loci implicated in the survival of Mycobacterium smegmatis in human mononuclear phagocytes. Mol Microbiol 29: 465-475.

49. Iantomasi R, Sali M, Cascioferro A, Palucci I, Zumbo A, et al. (2011) PE_PGRS30 is required for the full virulence of Mycobacterium tuberculosis. Cell Microbiol 14: 356-367.

50. Grundner C, Cox JS, Alber T (2008) Protein tyrosine phosphatase PtpA is not required for Mycobacterium tuberculosis growth in mice. FEMS Microbiol Lett 287: 181-184.

51. Rengarajan J, Murphy E, Park A, Krone CL, Hett EC, et al. (2008) Mycobacterium tuberculosis Rv2224c modulates innate immune responses. Proc Natl Acad Sci U S A 105: 264-269.

52. Dahl JL, Wei J, Moulder JW, Laal S, Friedman RL (2001) Subcellular localization of the Iitracellular survival-enhancing Eis protein of Mycobacterium tuberculosis. Infect Immun 69: 4295-4302.

53. Singh A, Singh Y, Pine R, Shi L, Chandra R, et al. (2006) Protein kinase I of Mycobacterium tuberculosis: cellular localization and expression during infection of macrophage-like cells. Tuberculosis 86: 28-33.

54. Jacobs M, Togbe D, Fremond C, Samarina A, Allie N, et al. (2007) Tumor necrosis factor is critical to control tuberculosis infection. Microbes Infect 9: 623-628.

55. Roach DR, Briscoe H, Saunders B, France MP, Riminton S, et al. (2001) Secreted lymphotoxin-alpha is essential for the control of an intracellular bacterial infection. J Exp Med 193: 239-246.

56. Roach DR, Bean AG, Demangel C, France MP, Briscoe H, et al. (2002) TNF regulates chemokine induction essential for cell recruitment, granuloma formation, and clearance of mycobacterial infection. J Immunol 168: 4620 4627.

57. Lee JS, Song CH, Lim JH, Kim HJ, Park JK, et al. (2003) The production of tumour necrosis factor-alpha is decreased in peripheral blood mononuclear cells from multidrug-resistant tuberculosis patients following stimulation with the 30kDa antigen of Mycobacterium tuberculosis. Clin Exp Immunol 132: 443-449.

58. Jung SB, Yang CS, Lee JS, Shin AR, Jung SS, et al. (2006) The mycobacterial 38-kilodalton glycolipoprotein antigen activates the mitogen-activated protein kinase pathway and release of proinflammatory cytokines through Toll-like receptors 2 and 4 in human monocytes. Infect Immun 74: 2686-2696.

59. Kim KH, Yang CS, Shin AR, Jeon SR, Park JK, et al. (2011) Mycobacterial heparin-binding hemagglutinin antigen activates inflammatory responses through PI3-K/Akt, NF-kappaB, and MAPK pathways. Immune Netw 11: $123-133$.

60. Byrd TF (1997) Tumor necrosis factor alpha (TNFalpha) promotes growth of virulent Mycobacterium tuberculosis in human monocytes iron-mediated growth suppression is correlated with decreased release of TNFalpha from iron-treated infected monocytes. J Clin Invest 99: 2518-2529.

61. Silver RF, Li Q Boom WH, Ellner JJ (1998) Lymphocyte-dependent inhibition of growth of virulent Mycobacterium tuberculosis $\mathrm{H} 37 \mathrm{Rv}$ within human monocytes: requirement for $\mathrm{CD} 4^{+} \mathrm{T}$ cells in purified protein derivative-positive, but not in purified protein derivative-negative subjects. J Immunol 160: 2408-2417.

62. Engele M, Stossel LE, Castiglione K, Schwerdtner N, Wagner M, et al. (2002) Induction of TNF in human alveolar macrophages as a potential evasion mechanism of virulent Mycobacterium tuberculosis. J Immunol 168: 1328-1337. 